\title{
Safety and efficacy of low-dose sirolimus in the PIK3CA- related overgrowth spectrum
}

Victoria E. R. Parker, MBBS PhD ${ }^{1}$, Kim M. Keppler-Noreuil, MD², Laurence Faivre, MD-PhD ${ }^{3}$, Maxime Luu, MD ${ }^{4}$, Neal L. Oden, $\mathrm{PhD}^{5}$, Leena De Silva, $\mathrm{BSc}^{1}$, Julie C. Sapp, $\mathrm{ScM}^{2}$, Katrina Andrews, MBChir ${ }^{1}$, Marc Bardou, MD-PhD ${ }^{4}$, Kong Y. Chen, $\mathrm{PhD}^{6}$, Thomas N. Darling, MD, PhD7, Elodie Gautier, MSc ${ }^{3}$, Barry R. Goldspiel, PharmD ${ }^{8}$, Smail Hadj-Rabia, MD-PhD ${ }^{9}$, Julie Harris, RN ${ }^{1}$, Georgios Kounidas, MPhil ${ }^{1}$, Parag Kumar, PharmD ${ }^{8}$, Marjorie J. Lindhurst, PhD ${ }^{2}$, Romaric Loffroy, MD-PhD ${ }^{10}$, Ludovic Martin, MD-PhD ${ }^{11}$, Alice Phan, MD-PhD ${ }^{12}$, Kristina I. Rother, MD ${ }^{6}$, Brigitte C. Widemann, MD ${ }^{13}$, Pamela L. Wolters, PhD ${ }^{13}$, Christine Coubes, MD ${ }^{14}$, Lucile Pinson, MD ${ }^{14}$, Marjolaine Willems, MD-PhD ${ }^{14}$, Catherine Vincent-Delorme, MD ${ }^{15}$ PROMISE Working Group\#, Pierre Vabres, MD-PhD ${ }^{3}$, Robert K. Semple, MBChir PhD ${ }^{1,16}$ and Leslie G. Biesecker, MD²

\begin{abstract}
Purpose: PIK3CA-related overgrowth spectrum (PROS) encompasses a range of debilitating conditions defined by asymmetric overgrowth caused by mosaic activating PIK3CA variants. PIK3CA encodes the p110a catalytic subunit of phosphatidylinositol-3kinase (PI3K), a critical transducer of growth factor signaling. As mTOR mediates the growth-promoting actions of PI3K, we hypothesized that the mTOR inhibitor sirolimus would slow pathological overgrowth.
\end{abstract}

Methods: Thirty-nine participants with PROS and progressive overgrowth were enrolled into open-label studies across three centers, and results were pooled. For the primary outcome, tissue volumes at affected and unaffected sites were measured by dual energy X-ray absorptiometry during 26 weeks of untreated run-in and 26 weeks of sirolimus therapy.

Results: Thirty participants completed the study. Sirolimus led to a change in mean percentage total tissue volume of $-7.2 \%$ (SD 16.0, $p=0.04)$ at affected sites, but not at unaffected sites $(+1.7 \%$, SD $11.5, p=0.48) \quad(n=23$ evaluable). Twenty-eight of $39(72 \%)$ participants had $\geq 1$ adverse event related to sirolimus of which $37 \%$ were grade 3 or 4 in severity and $7 / 39$ (18\%) participants were withdrawn consequently.

Conclusion: This study suggests that low-dose sirolimus can modestly reduce overgrowth, but cautions that the side-effect profile is significant, mandating individualized risk-benefit evaluations for sirolimus treatment in PROS.

Genetics in Medicine (2019) 21:1189-1198; https://doi.org/10.1038/s41436018-0297-9

Keywords: overgrowth; mosaicism; PIK3CA; sirolimus

\section{INTRODUCTION}

PIK3CA-related overgrowth spectrum (PROS) designates a heterogeneous group of rare, asymmetric overgrowth disorders caused by postzygotic variants in the gene PIK3CA. ${ }^{1}$ PIK3CA encodes the p110a catalytic subunit of phosphoinositide 3-kinase (PI3K), which transduces activation of tyrosine kinase growth factor and hormone receptors into activation of AKT and mTOR signaling ${ }^{2}$ to promote tissue growth. PIK3CA variants in PROS cause physiologically inappropriate activation of $\mathrm{AKT}$ and mTOR, and variable, asymmetric overgrowth, consistent with causation by an early developmental stochastic pathogenic variant. Overgrowth includes adipose tissue, muscle, skin, bone, blood or lymph vessels, or neural tissue, among others. ${ }^{3-6}$ Adipose and vascular components are particularly striking, reflecting the inherent plasticity and postnatal growth potential of these tissues. Complications of PROS depend on the anatomical site and extent of overgrowth, but may include functional impairment (e.g., of walking or swallowing), pain, recurrent superficial infections, thromboembolism, and/or hemorrhage, all of which may be debilitating, and cause early mortality. Current treatment is inadequate, relying on debulking

Correspondence: Robert K. Semple (rsemple@ed.ac.uk). "Affiliations are listed at the end of the paper.

These coauthors contributed equally: Victoria E.R. Parker, Kim M. Keppler-Noreuil, and Laurence Faivre.

These senior coauthors contributed equally: Pierre Vabres, Robert K. Semple, and Leslie G. Biesecker.

\#List of authors in the PROMISE Working Group is given in the supplementary material.

Submitted 19 April 2018; accepted: 29 August 2018

Published online: 1 October 2018 
surgery, amputation, and/or endovascular occlusive procedures. Regrowth following surgery occurs frequently and repeated surgery is common.?

Some genotype-phenotype correlation in PROS has been suggested, ${ }^{8,9}$ however the dominant determinant of phenotype is the timing and location of the pathogenic variant. This causes a high degree of interindividual phenotypic heterogeneity in PROS. Compounding this, growth trajectories vary greatly for unknown reasons, with some exhibiting excess growth limited to childhood, while others have progressive soft tissue overgrowth during adult life. Finally, overgrown tissue usually lacks the clear anatomical demarcation characteristic of neoplasia on imaging. Collectively these considerations complicate the design of quantitative endpoints for therapeutic studies.

Allosteric mTOR inhibitors such as sirolimus are approved for posttransplant immunosuppression. They can also slow the growth of cancers bearing PIK3CA variants. ${ }^{10}$ Sirolimus potently attenuates pathological AKT signaling and reduces cell proliferation in dermal fibroblasts derived from people with PROS, ${ }^{11,12}$ which suggests that it could be an effective treatment of PROS. Anecdotal reports of sirolimus therapy in PROS have suggested efficacy, ${ }^{13,14}$ while other studies have reported clinical improvement in patients with vascular overgrowth caused by TIE2 variants ${ }^{15}$ or of unknown cause. ${ }^{16,17}$ Further, unpublished patient and clinician reports suggest wider off-label use of sirolimus in PROS. To assess the potential efficacy of sirolimus in PROS further, and to address the challenges of study design, we undertook a study of lowdose sirolimus in patients with molecularly proven PROS, assessing quantitative disease endpoints. The study was conducted across three centers using a common protocol.

\section{MATERIALS AND METHODS}

This pilot study was performed in accordance with the Declaration of Helsinki and Good Clinical Practice guidelines or the US human subject research regulations (US Code of regulations 45CFR46). The study protocol was approved by ethics review boards of each study site. Regulatory approval from the Medicines and Healthcare Products Regulatory Agency (MHRA) was obtained in the United Kingdom, and Agence Nationale de Sécurité du Médicament et des Produits de Santé (ANSM) in France. Written informed consent and/ or parental consent and where possible assent for the participation of children was obtained for all participants. Study Registration: France (NCT02443818), United Kingdom (EudraCT: 2014-000484-41), United States (NCT02428296).

\section{Study overview and design}

We selected a nonrandomized, open-label design with a 26week observational run-in period to measure growth (Fig. 1a). The study was conducted at Cambridge University Hospitals NHS Trust (United Kingdom), CHU Dijon-Bourgogne (France), and the National Human Genome Research Institute (United States) (Supplemental Table S1). Intraparticipant comparisons between affected and matched unaffected contralateral tissue were made where possible. A placebo was excluded pragmatically due to growing awareness of the availability of sirolimus and its increasing off-license use in PROS, potentially compromising recruitment within this orphan disease population. Participants were assessed for sirolimus efficacy at week 0 (before the run-in phase), week 26 (after the run-in phase and before treatment), and at week 52 (after 26 weeks of treatment).

\section{Inclusion/exclusion and withdrawal criteria}

Eligible participants were aged from 3 to 65 years, inclusive. All had a diagnosis of PIK3CA-related overgrowth spectrum $(\mathrm{PROS})^{9,18}$ with progressive overgrowth (determined by selfreport or serial measurements) and a mosaic PIK3CA variant identified by methodology described previously. ${ }^{5,9,18}$ (Table $\mathbf{1}$ ). The main exclusion criteria were use of sirolimus in the prior 4 weeks; active skin infections; major surgery in the prior 12 weeks; pregnancy and lactation; live vaccines in the 4 weeks prior to, during, and 6 weeks after dosing; insufficient renal, hepatic, or bone marrow function; or prior/intercurrent HIV, hepatitis $\mathrm{B} / \mathrm{C}$, or tuberculosis infection. In the United Kingdom and France, participants with a blood neutrophil count of $<1.0 \times 10^{9} / 1$ were excluded; in the United States, participants with a count of $<1.5 \times 10^{9} / 1$, or $<1.0 \times 10^{9} / 1$ and a diagnosis of benign ethnic neutropenia were excluded.

Participants were withdrawn if they or their parents requested, or if adverse events (AEs) of grade $\geq 3$ occurred including neutropenia $\left(<1.0 \times 10^{9} / 1\right.$ in France and the United Kingdom, $<0.5 \times 10^{9} / 1$ in the United States); proteinuria (e.g., $3+$ on qualitative testing); liver dysfunction (alanine transaminase $[\mathrm{ALT}]>3$ times the upper limit of normal); renal dysfunction (glomerular filtration rate [GFR] $<70 \mathrm{mls} / \mathrm{min} /$ $1.73 \mathrm{~m}^{2}$ ); pneumonitis or decline in respiratory reserve; severe, recurrent infections or septicemia; QTc prolongation; or other side effects at the discretion of the lead investigator at each site.

\section{Dosing regimen}

Pharmacokinetic data for sirolimus for children and adults with renal transplants informed dosing algorithms. ${ }^{19}$ A target sirolimus plasma concentration of $2-6 \mathrm{ng} / \mathrm{ml}$ was selected based on in vitro preclinical studies ${ }^{20}$ off-label clinical experience, ${ }^{13}$ and with the aim of minimizing AEs. Given the known immunosuppressive effects of high-dose sirolimus, the lack of evidence that these doses are more efficacious in PROS, and the likely need for lifelong therapy we titrated dosing to achieve this target concentration. Steady state was defined as two consecutive concentrations within $10 \%$ of each other. Dosing and sirolimus monitoring regimens between the UK, France, and US sites are outlined in Supplemental Table S5. Treatment was paused during intercurrent infection, surgical intervention, or other $\mathrm{AE}$ when necessary.

\section{Biochemical evaluation}

Laboratory assessments included blood cell counts, indices of renal function and liver function, fasting blood glucose and 


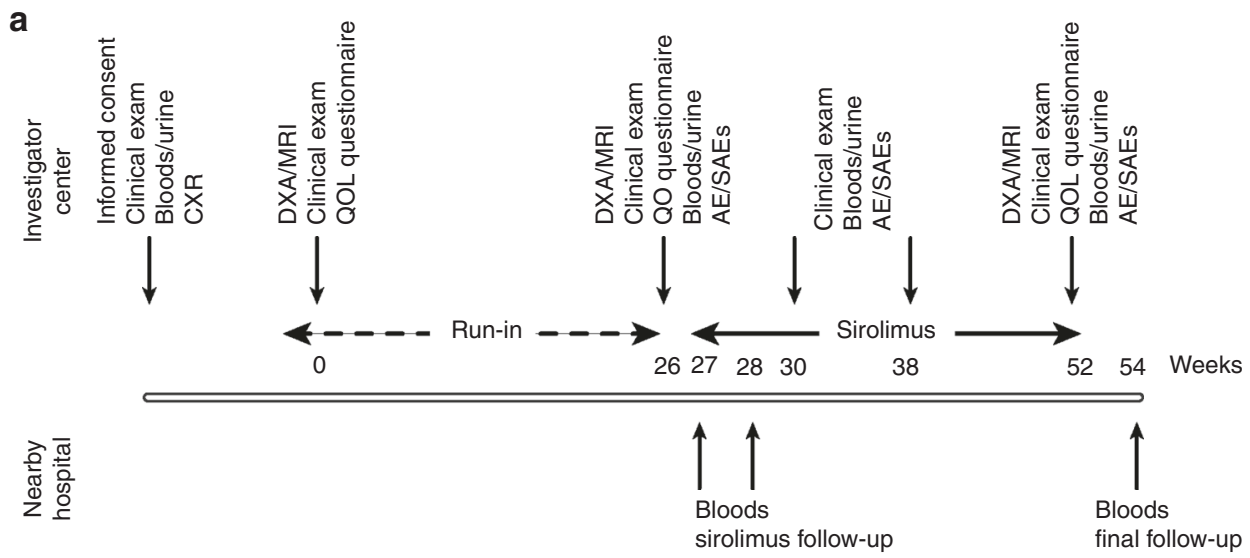

b

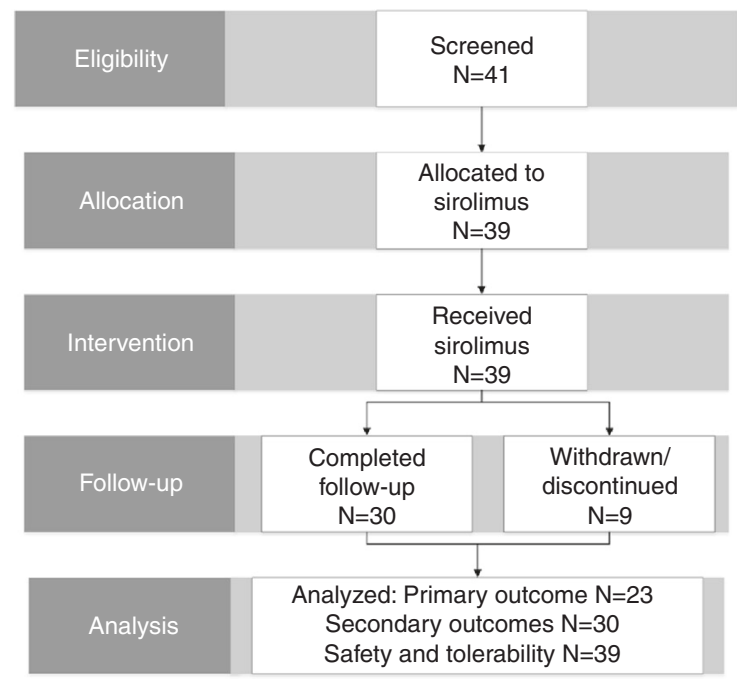

Fig. 1 CONSORT flow-chart and schematic of nonrandomized open-label pilot study. a Schematic of number of participants assessed for eligibility and excluded or allocated to the study, treated, followed, and analyzed. Of the 39 subjects enrolled, 30 completed 26 weeks of sirolimus therapy, and 23/30 had anatomy that permitted analysis of the primary outcome measure. Safety and tolerability were evaluated in all treated participants. $\mathbf{b}$ Overview of study design including schedule of procedures. AE/SAES adverse events/serious adverse events, CXR chest X-ray, DXA/MRI dual energy X-ray absorptiometry/ magnetic resonance imaging, QOL quality of life.

lipid profiles, and urinalysis including protein semiquantification. Sirolimus assays were performed in accredited clinical diagnostic laboratories.

\section{Quantification of overgrowth}

Affected sites were defined by clinical observation. Unaffected sites showed no overgrowth, nor evidence of skin or vascular abnormalities. Unaffected sites compared with affected sites were (in order of preference) the contralateral limb/truncal region, a limb or trunk on the same side, or any other site without clear involvement. Those with generalized, but asymmetric overgrowth were deemed to have no unaffected site.

Dual energy X-ray absorptiometry (DXA) scans were performed using the same orientation and iDXA GE Lunar scanner for each participant at 0,26 , and 52-week time points. DXA imaging provides reproducible measurement of regional and whole body composition (bone, fat, and lean mass), ${ }^{21}$ though it cannot assess vascular or lymphatic malformations.
We used DXA to evaluate the primary outcome measure in view of its wide applicability, its acceptability to children, and its capacity to generate concurrent estimates of unaffected and affected tissue sites. For adults too large to scan completely with one scan $(n=3)$, a second scan was performed to give full body coverage. Soft tissue volumes were obtained for total body and various body segments (left leg, right leg, right trunk, left trunk, right arm, left arm, and head) by converting masses to volumes assuming fat density of $0.9 \mathrm{~g} / \mathrm{ml}$ and lean mass density of $1.1 \mathrm{~g} / \mathrm{ml} .^{22}$ Total tissue volume included lean and fat volume, but not bone. Results were generated using the same, standardized iDXA GE Lunar software at each center.

T1-weighted magnetic resonance imaging (MRI) scans without contrast were also acquired in a subset of participants at 0,26 , and 52 weeks using the same scanner at each site (GE GEMR450 wide in the United Kingdom, IRM Siemens Magnetom Aera 1.5T in France, and 3T MRI scanner [Trio, Siemens Medical Solutions] in the United States). Scanning 
Table 1 Baseline characteristics of enrolled participants

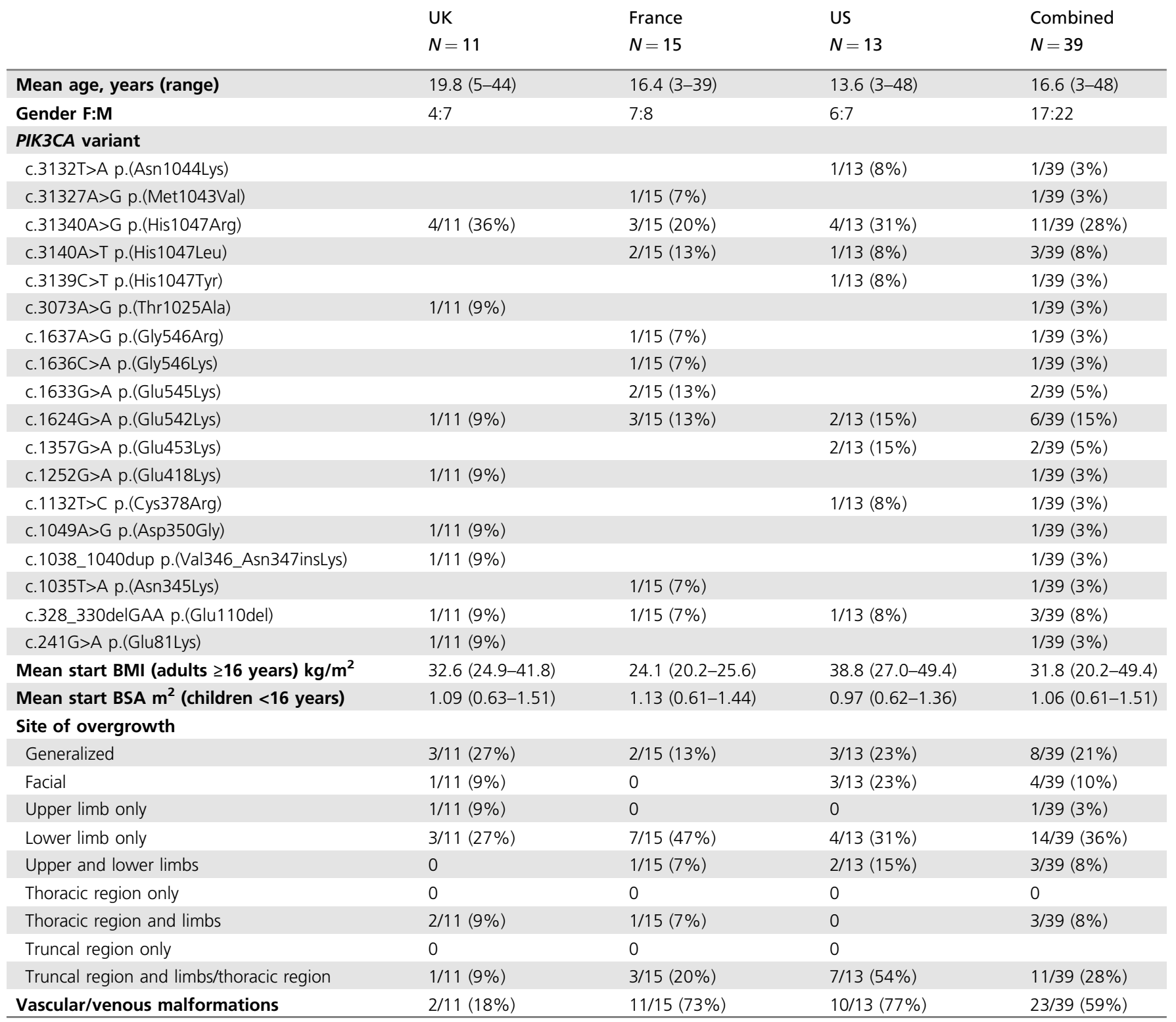

$B M I$ body mass index, BSA Body Surface Area

covered bony anatomical landmarks at proximal and distal ends of the target area, and an oblique scan plane of $5 \mathrm{~mm}$ thickness with up to 100 slices was used. All scans were blinded prior to analysis. For volume calculation, IDEAL fat (Dixon sequence) images were visualized using volumetric software (SliceOmatic, TomoVision, Magog, Canada). Morphology segmentation was performed through computation of watershed gradients. Tissues (fat, muscle, bone, and blood vessel) were manually defined and software was used to generate a surrogate of tissue volume using five slices, with manual adjustments where required.

\section{Evaluation of vascular lesions}

Clinical photography was performed in participants with visible vascular lesions before and after sirolimus treatment using the same camera in the same room with consistent lighting and color balance. Unblinded assessment by a study investigator was undertaken using the following scoring system: $-1=$ worsening; $0=$ no change; $1=$ partial resolution; $2=$ complete resolution. Vascular lesions were scored by hue, with annotation regarding the presence of lymphatic blebs.

\section{Quality of life assessment}

Validated quality of life (QOL) questionnaires were administered before and after treatment (WHOQOL-BREF questionnaire for adults, $^{23}$ and age-appropriate PedsQL questionnaires for children and parents ${ }^{24,25}$ ). The WHOQOL-BREF assesses four domains (physical, psychological, social, environmental). ${ }^{26,27}$ The PedsQL Generic Core 
Scales assess four domains (physical, emotional, social, and school) and two composite scores (physical, psychosocial), which were transformed to a $0-100$ scale (25).

\section{Outcome measures}

The primary outcome measure was percentage change in volume of measured affected and unaffected areas over treated and untreated periods. Fat, lean, and total (fat plus lean) tissue volumes were determined. Both DXA and volumetric MRI scanning were undertaken where possible and anatomically appropriate. Prior to analysis, DXA was selected for the primary outcome measure as DXA results were available in all participants whereas MRI was only performed in a subset. Secondary outcome measures were steady-state sirolimus plasma concentration, mean sirolimus doses to achieve the target plasma concentration, and additional measures of efficacy including hospitalizations, surgical interventions, and QOL scores before and after treatment.

\section{Statistical analyses}

Intention-to-treat analyses were based on all enrolled participants. As-treated analyses were restricted to those who completed the study. Absolute volumes of affected and unaffected tissue at week 0 (designated $\mathrm{X}$ ), week 26 (designated $\mathrm{Y}$ ), and week 52 (designated $\mathrm{Z}$ ), were compared. Tissue volume changes (week 0-26 and week 26-52) were designated "DELTA," and the percent change "\% Change." Percent change for the untreated period was $[100(Y-X / X)]$, and for the treated period $[100(\mathrm{Z}-\mathrm{Y} / \mathrm{Y})]$. Paired comparisons of mean volumes and mean changes in volumes during untreated ad treated periods were performed using singlesample, paired Student's $t$ test in SAS version 9.4, with confirmation of equal variances. A post hoc subanalysis was performed to examine the difference $\%$ change in tissue at affected and unaffected sites during the treatment phase in comparison with the run-in phase according to age, genotype, and phenotypic characteristics. Linear regression and analysis of variance (ANOVA) analyses were performed to evaluate statistical significance. Additional statistical analyses of normally distributed data of equal variance were performed using single-sample Student's paired $t$ tests and chi-squared analyses for discontinuous data.

\section{Safety analysis}

Safety endpoints were adverse events (AEs) identified by laboratory testing, clinical examination, or self-report. Severity was graded with the Common Terminology Criteria for Adverse Events (CTCAE) (version 4.0). AEs were coded by the current version of the Medical Dictionary for Regulatory Activities (MedDRA), and the type, incidence, severity, and relationship to sirolimus were summarized by MedDRA System Organ Class and Preferred Term. Serious adverse events (SAEs) were defined as those that led to death, were lifethreatening, necessitated hospital admission or prolongation of an admission, or that resulted in persistent or severe disability or incapacity, congenital anomalies, or any other medically important events. All AEs and SAEs possibly, probably, or definitely related to sirolimus were reviewed by an international committee composed of members of the study teams from the United Kingdom, France, and the United States.

\section{RESULTS \\ Study participants and disposition}

Forty-one participants were screened and 39 enrolled, including 22 children $(<16$ years old) and 17 adults $(\geq 16$ years old) (Fig. 1b). The mean age was 16.6 years (SD 11.0, range 3-48); $44 \%$ were female and 56\% male. Clinical characteristics of these participants are detailed in Supplementary Table S2, and representative images illustrating the overgrowth heterogeneity are shown in Fig. 2. Thirty participants completed 26 weeks of dosing, 2 discontinued the study after 23 weeks of dosing due to a temporary pause in dosing at one of the sites, and 7 were withdrawn due to AEs (Fig. 1b) (Supplementary Table S3). Of the 30 who completed 26 weeks dosing, $97 \%$ received $>80 \%$ of their daily doses.

\section{Sirolimus plasma levels and dosing}

The mean plasma sirolimus level recorded across the study in both adults and children was $3.4 \mathrm{ng} / \mathrm{ml}$ (95\% confidence interval [CI] 3.1, 3.7), and the median level $3.3 \mathrm{ng} / \mathrm{ml}(25 \mathrm{th} /$ 75 th centiles $2.3,4.2)$. The mean total daily sirolimus dose in adults was $1.2 \mathrm{mg}$ once daily in capsules (95\% CI 1.1, 1.3), and for children was $0.58 \mathrm{mg}$ twice daily in oral solution (95\% CI $0.50,0.65)$.

\section{Efficacy-based outcome measures}

Volumes of fat and lean components of affected and (where possible) unaffected soft tissue body regions were measured using DXA at 0,26 , and 52 weeks (Fig. 3a). The anatomy of 23 participants permitted comparison of affected versus unaffected. Absolute tissue volumes were greater and more variable at affected versus unaffected sites as expected; at week 0 median total tissue volume at affected sites was $10,132 \mathrm{ml}$ (interquartile range [IQR]: 4980, 17,055) and $3013 \mathrm{ml}$ (IQR: $1465,8599)$ at unaffected sites. In the untreated run-in period, the mean increase in total tissue volume at affected sites was $+7.9 \%$ (SD 12.8) versus $+4.8 \%$ (SD 9.7) at unaffected sites, $(p=0.19$; SD 10.9) (Fig. 3b).

For the primary outcome measure, a significant reduction of $-7.2 \%$ (SD 16.0; $p=0.04$ ) was observed in the volume change of affected tissues during sirolimus treatment. In contrast, there was no significant increase for unaffected tissue $(+1.7 \%$, SD $11.5 ; p=0.48)$. A significant reduction in lean tissue (muscle, connective tissue, and vasculature) of $-6.3 \%$ (SD 13.3; $p=0.03$ ) and a trend toward reduction of fat of $-9.8 \%$ (SD 24.4; $p=0.07$ ) was observed at affected but not unaffected sites during treatment (Supplementary Table S4). Viewing individual data points on scatter plots comparing volume changes in treated and untreated phases reinforces this conclusion (Supplementary Figure S1).

A post hoc subanalysis was performed to assess differences in response by age, clinical phenotype (qualitatively 
predominant adipose overgrowth, vascular overgrowth, or muscular/bony overgrowth), genotype, or overgrowth rate during the run-in period (Supplementary Figure S2). A significant correlation between the magnitude of treatment effect (difference between tissue volume changes in treatment and run-in periods) and of progressive overgrowth during the run-in period was seen (Fig. 3c). In addition, there was a trend toward greater tissue volume reduction in participants with predominant adipose overgrowth (-8.9\% [SD 14.9], $n=$ $17 / 23)$ than in participants without predominant adipose overgrowth $(-2.4 \%$ [SD 19.4], $n=6 / 23, p=0.14)$. There were no apparent differences with respect to age, genotype, or the presence/absence of vascular/venous malformations.

Serial MRI analyses of tissue volume were available for only $10 / 20$ participants due to the site of overgrowth not being amenable to MRI scanning, or due to insufficient quality of a

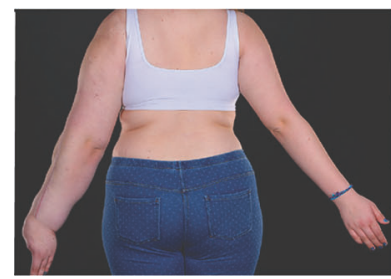

e

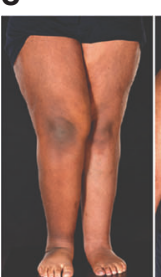

b

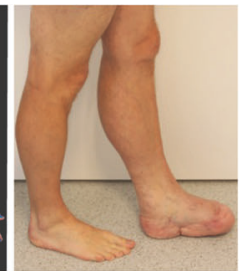

c

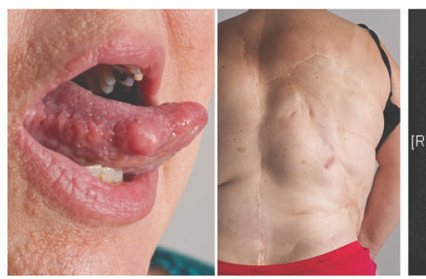

d

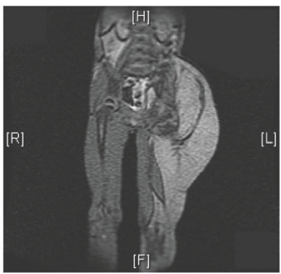

g
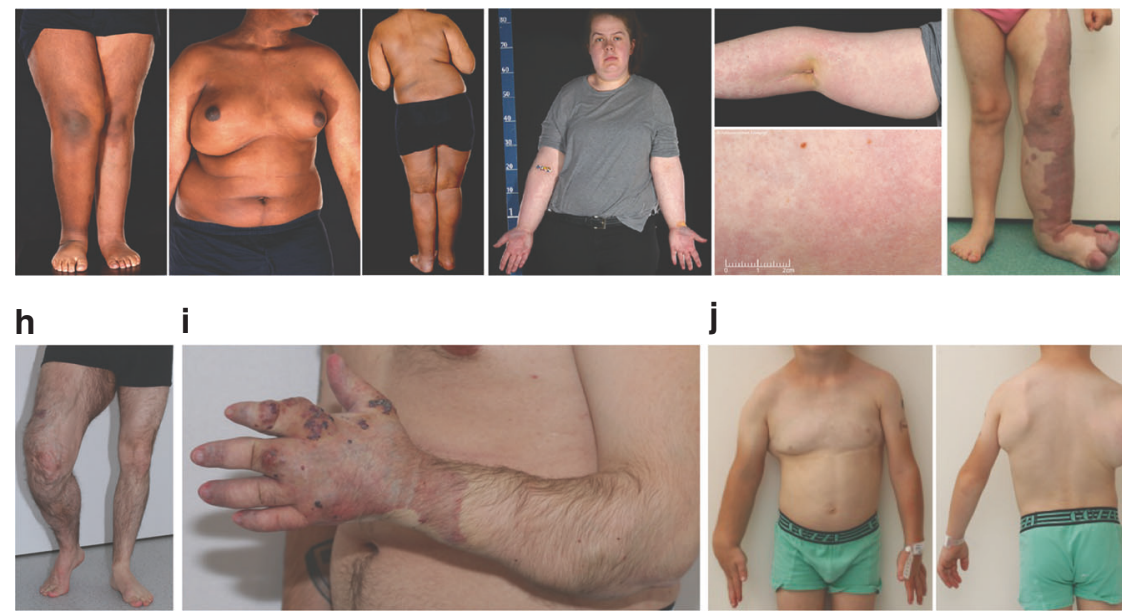

i
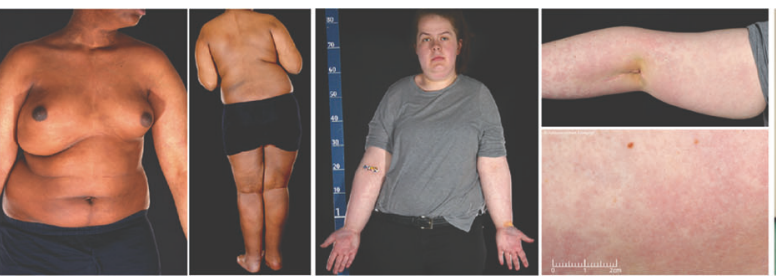

j
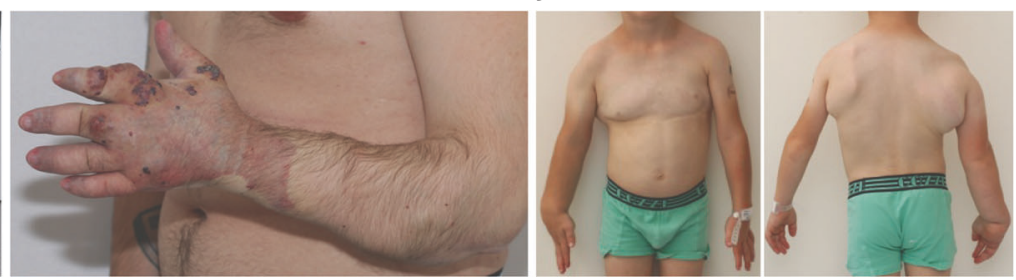

k

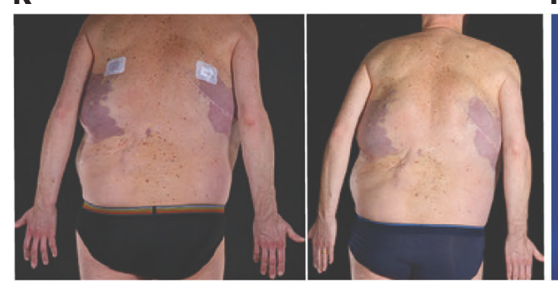

I

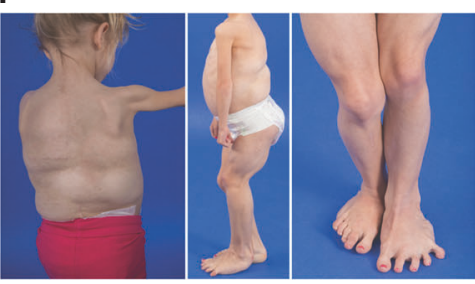

m

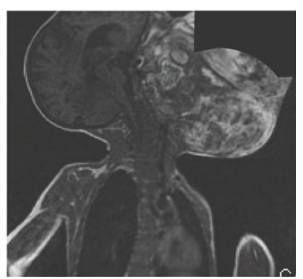

n

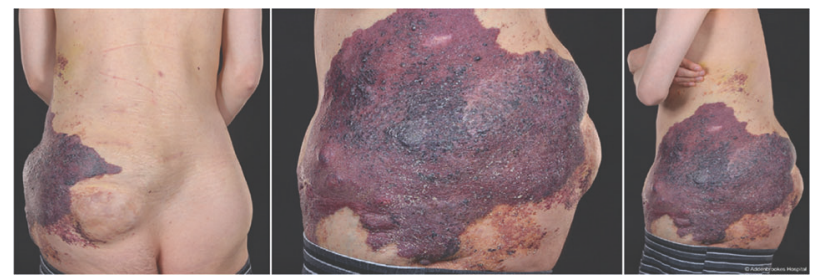

o

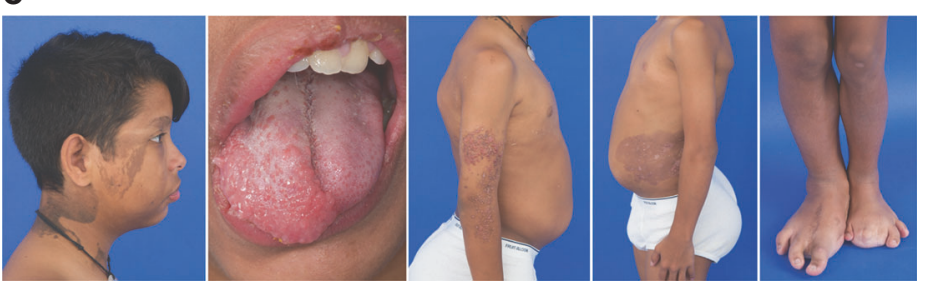


acquired images for analysis. Technical challenges included variation in participant position and movement, varying slice thickness between scans, and anatomical factors such as fat investiture into muscles, preventing clear definition of tissue planes (Supplementary Figure S3). Only six of ten MRI series that were eligible for analysis included both affected and unaffected sites. No statistically significant differences in tissue volume changes between the treatment phase and runin phase were seen for either affected sites $(-3.7 \%$ [SD 12.3]; $p=0.37$ ) or unaffected sites (0\% [SD 16.7]; $p=0.99)$.

\section{Secondary outcome measures}

No differences were detected in QOL scores before and after sirolimus treatment (Supplementary Table S5) among adults or children. During run-in, five hospitalizations in five participants and two surgical interventions in two participants were recorded. In the treatment phase 15 hospitalizations in 9 participants and no surgical interventions arose. This difference was not significant $(p=0.24)$. Clinical photographs were evaluated for eight participants with superficial capillary or venous malformations and none changed during treatment. Five participants had lymphatic malformations and of these, two showed evidence of improvement with decreased superficial lymphangiectasis (Supplementary Figure S4), with three unchanged.

\section{Safety and tolerability}

Twenty-eight of 39 participants (72\%) had at least one AE deemed possibly, probably, or definitely related to sirolimus (Table 2). Twenty-one serious AEs (SAEs) (Supplementary Table S6) occurred among 12 participants during either runin or treatment periods, and 7 had AEs leading to sirolimus discontinuation. The most common class of AE was infection (16/39 [41\%] participants), followed by blood or lymphatic disorders (8/39 [21\%]) (Supplementary Table S7). Clinically important AEs included grade 4 neutropenia (neutrophil count $0.02 \times 10^{9} / \mathrm{L}$ ), interstitial pneumonitis, and sirolimus hypersensitivity syndrome. This last SAE presented with prolonged fever and resulted in hospitalization for more than 6 weeks. Sirolimus was withdrawn in all three participants, all of whom subsequently made full recoveries. There were no significant changes in laboratory assessments including lipid profiles and fasting glucose concentrations in the treatment period.

\section{DISCUSSION}

In this study 26 weeks of low-dose sirolimus therapy was associated with a small, but significant reduction in tissue growth at overgrown sites in participants with PROS. There was no measured effect on QOL or the overall extent of PROS-related skin lesions. These findings bolster the notion that sirolimus may be beneficial in PROS. One aim of this study was to inform selection of endpoints, and the powering and design of such trials.

Overgrowth in PROS comprises admixture of adipose tissue, vascular tissue, and bone. We anticipated that adipose and vascular tissues would be the most likely to respond to short-term treatment, given their natural capacity for rapid growth in the face of positive energy balance or injury. Furthermore, as these tissues can also regress physiologically, and as PIK3CA is involved in both cell proliferation and suppression of apoptosis, shrinkage of previously overgrown tissue rather than just deceleration of pathological overgrowth seemed a conceivable outcome of therapy. Using DXA body composition analysis we did not document evidence of significant and selective regression of affected tissue; however, overgrowth during run-in was associated with greater reduction in tissue volume during treatment. We also set out to use volumetric MRI to quantify adipose tissue volumes; however, technical and analytic challenges and inconsistency in serial imaging compromised this. MRI may nevertheless still be useful with more rigorous control of positioning and slice thickness.

This study had several limitations. Despite genetic homogeneity, phenotypic heterogeneity among participants was considerable. Post hoc subanalyses based on phenotype were inconclusive due to small sample size. Formally establishing whether our findings are generalizable to all PROS components, including the minor end of the phenotypic spectrum, will require availability of large PROS cohorts in future studies. The high frequency of study visits during treatment and the nonrandomized, open-label design may have led to

Fig. 2 Clinical heterogeneity of study participants with PIK3CA-related overgrowth spectrum. a 15-year-old girl with fibroadipose hyperplasia of left arm; unaffected right arm (patient 1). b 39-year-old man with fibroadipose and bony hyperplasia of left lower leg, foot s/p multiple amputations, and lipectomies; unaffected right leg, foot (patient 17). c 48-year-old woman with tongue fibromas, fibroadipose hyperplasia masses of trunk, back, paraspinal s/ $\mathrm{p}$ (status post) excision, and scoliosis s/p surgical bracing (patient 30). d 5-year-old boy with fibroadipose hyperplasia of the left leg, buttock visualized on magnetic resonance image (MRI) scan (patient 4). e 17-year-old boy with right fibroadipose hemihyperplasia (patient 2). f 19-year-old woman with right fibroadipose hemihyperplasia and capillary malformation, MCAP (Megalencephaly-capillary malformation syndrome) phenotype (patient 3). g 6-year-old girl with vascular malformations/overgrowth of left leg, foot; unaffected right leg, foot (patient 19). $\mathbf{h}$ 26-year-old man with vascular malformations/overgrowth of right leg; unaffected left leg (patient 12). i 23-year-old man with vascular malformations of the left arm and hand (patient 13). $\mathbf{j} 3$-year-old boy with fibroadipose hyperplasia of the right arm, hand, fingers, and trunk and left partial chest/axilla (patient 16). $\mathbf{k}$ 44-year-old man with fibroadipose hyperplasia and vascular malformations of his back and bilateral legs (patient 10). I 8-year-old girl with fibroadipose hyperplasia masses of her trunk, back, paraspinal s/p excision, muscular hyperplasia of her legs and feet (left > right), regional lipohypoplasia of her bilateral arms (patient 27). $\mathbf{m}$ 6-year-old boy with facial infiltrating lipomatosis affecting left cheek visualized on MRI scan (patient 9). $\mathbf{n} 31$-year-old man with vascular malformations and fibroadipose hyperplasia of the lower trunk, leg (patient 5). o 13-year-old boy with epidermal nevus of right face, neck; right facial, tongue, trunk, bilateral legs, feet (right >left) fibroadipose hyperplasia s/p multiple amputations/debulking surgeries of feet and toes; lymphovascular malformations of bilateral arms (right $>$ left), trunk (patient 29). Written informed consent was obtained for use of all identifiable images. 
a

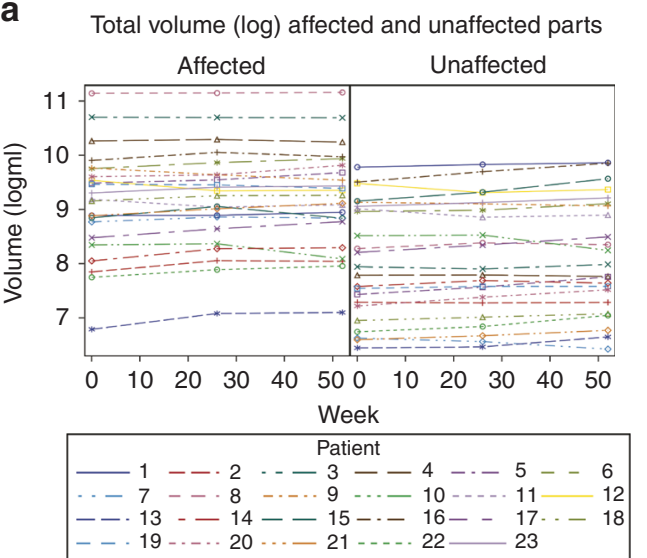

c

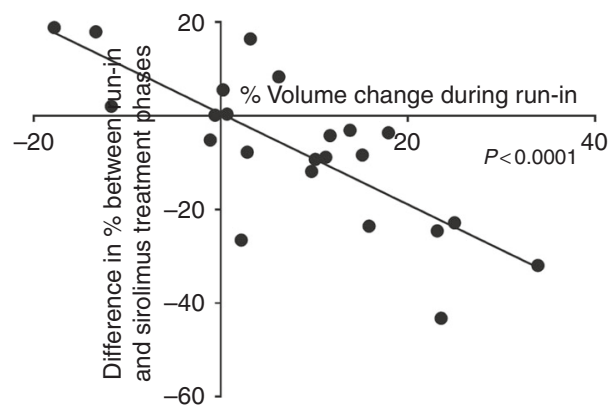

b

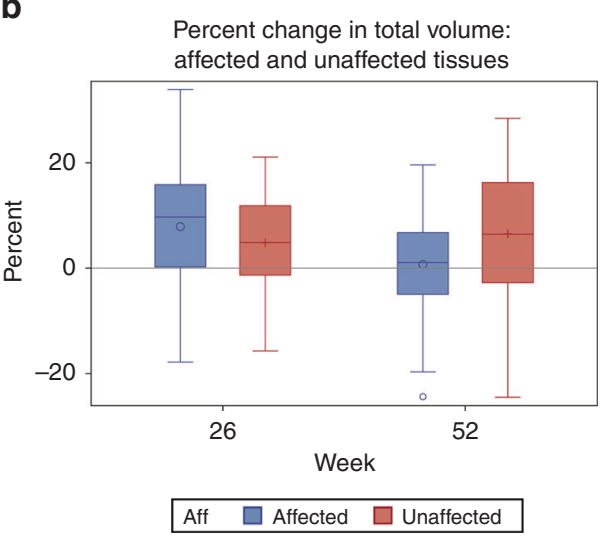

Fig. 3 Dual energy X-ray absorptiometry (DXA)-estimated changes in tissue volume during study period. a Change in absolute volumes in ml at affected and unaffected sites at the beginning of the run-in period (week 0), after the 26-week run-in period (week 26), and after the 26-week sirolimus treatment period (week 52). Note major differences in the magnitude and variability of volume measurements between affected and unaffected sites. b Change in percent tissue volume at affected and unaffected sites during the run-in period at week 26 and during the sirolimus treatment phase at week 52. Individual values plotted, bars denote mean values and error bars standard deviation. c Post hoc subanalysis of progressive growth during the run-in period. Linear regression analysis of \% volume change during run-in period, i.e., whether or not subjects had progressive overgrowth versus difference in \% volume change between the run-in and treatment phase at affected sites. The result was significant implying the largest effect sizes were obtained in subjects with progressive overgrowth during the run-in period.

Table 2 Overview of adverse events (AEs) and serious adverse events (SAEs)

Intention-to-treat

population $(n=39)$

\begin{tabular}{ll}
\hline Total number of AEs recorded & $\begin{array}{l}103 \text { AEs in 31/39 (79\%) } \\
\text { participants } \\
54 / 103 \text { AEs (52\%) } \\
\text { unrelated to sirolimus }\end{array}$ \\
& $28 / 39(72 \%)$ \\
\hline At least one sirolimus-related AE (all grades) & $13 / 39(33 \%)$ \\
\hline At least one event of $\geq$ grade 3 severity & $0(0 \%)$ \\
\hline Death (grade 5) & $11 / 39(28 \%)$ \\
\hline At least one SAE & $10 / 39(26 \%)$ \\
\hline At least one SAE and $\geq$ grade 3 severity & $7 / 39(18 \%)$ \\
\hline $\begin{array}{l}\text { At least one event leading to permanent } \\
\text { discontinuation of sirolimus }\end{array}$ & \\
\hline
\end{tabular}

overestimation of AEs. Furthermore, the time taken for sirolimus plasma concentrations to reach steady state and interruption of treatment due to intercurrent AEs in some patients will have reduced drug exposure and thus any treatment effect. The QOL assessment was limited by small sample sizes, and use of a generic assessment tool that omitted pain and functional impairment scoring. Additional tools exist, but none are specifically tailored to the PROS spectrum. Finally, participants who had generalized but asymmetric overgrowth were not included in the primary outcome analysis as an unaffected site could not be identified. In other patients, the "affected" and "unaffected" body regions we studied were defined based on clinical and radiographic appearances. However, the presence of variant-positive cells in apparently unaffected sites cannot be excluded, and thus growth at these sites could be at least partly pathologic.

Our findings suggest that, in the 26-week period assessed, sirolimus exerted its greatest effect on actively growing tissue, rather than inducing regression of prior overgrowth. Sirolimus did not significantly reduce tissue growth in unaffected areas, suggesting that sirolimus does not inhibit normal cellular growth at low doses. Subanalyses suggested a greater response in participants with predominantly adipose overgrowth; however, only the effect on lean tissue volume, assessed by DXA, reached significance. We cannot rule out greater effects of higher doses of sirolimus or longer durations of therapy; however, the effect size we observed will be of value in powering future studies. 
A recent study by Adams et al. ${ }^{17}$ assessed a higher dose of sirolimus in a heterogeneous cohort with primarily vascular malformations. No genetic characterization was reported, but some were likely to have PROS based on clinical phenotypes. In that study, disease response (complete or partial) was defined with respect to radiologic imaging or QOL measures. No participant had complete response, and $87 \%$ had partial response, in some cases due only to changes in QOL measures. That study had limitations of likely heterogeneous etiology, no control or run-in period was used, affected and unaffected sites were not compared, and various imaging modalities were employed.

Medical therapy for PROS is likely to be suppressive rather than curative, and may be indefinite from childhood. Mindful of this, and of evidence that low concentrations of mTOR inhibitors are sufficient ex vivo to suppress basal hyperactivation of PI3K in cells from people with PROS, ${ }^{12,20}$ we used low-dose sirolimus to attempt to reduce the $\mathrm{AE}$ rate reported with higher doses. This yielded plasma concentrations below the immunosuppressive range used after organ transplantation. Nevertheless, a considerable number of AEs, several severe, were recorded, leading to 7 discontinuations of treatment, although 11/39 (28\%) participants had no reported AEs. In comparison with Adams et al. ${ }^{17}$ we observed higher rates of discontinuation and infection-related AEs $(41 \%$ in this study versus $15 \%$ [ref. ${ }^{17}$ ] for CTCAE grade 2 or higher), but a lower incidence of hematological AEs (5\% versus 27\% [ref. ${ }^{17}$ ] for CTCAE grade 3 or higher). In contrast, an AE profile that similar to ours was reported in a study of everolimus in tuberous sclerosis. ${ }^{28}$ There, $11 / 28$ (39\%) participants had a $\geq$ grade 3 event (versus 13/39 [33\%] in our study) and the most frequent events were stomatitis $22 / 28$ (79\%), upper respiratory tract infection $22 / 28$ (79\%), and sinusitis $11 / 28(39 \%)$. Discontinuation rates were only $1 / 28$ (3.5\%) in the first 6 months in the tuberous sclerosis trial. It is unclear whether some of the reported differences relate to the biology of PROS, to the heterogeneity of the participants in the prior studies, or whether they reflect a greater risk of infection with sirolimus therapy in PROS. Immunodeficiency is seen in several germline disorders featuring activated PI3K signaling, but in many patients with PROS the variant allele fraction in blood is undetectable or extremely low. We conclude that use of low-dose sirolimus in this study did not demonstrably decrease $\mathrm{AE}$ rates seen with higher-dose therapy. $^{29}$

In summary, sirolimus has potential benefit for patients with PROS, especially where progressive adipose tissue overgrowth predominates. Randomized controlled trials with designs optimized for rare disease research are required to confirm this, and to establish the optimal therapeutic window, especially given our observed high rate of discontinuations despite the use of low-dose treatment. Based upon these results, sirolimus use in PROS should be evaluated on a caseby-case basis. Our study holds lessons for design of future trials, whether of mTOR inhibitors, or of trial agents targeting the PIK3CA gene product directly. The importance of objectively establishing the efficacy of sirolimus for PROS assumed new urgency while this study was in review, with the reporting of an uncontrolled, unregistered case series of patients with PROS treated with an experimental PIK3CA inhibitor on a compassionate basis. ${ }^{30}$ These anecdotal data suggested clinical efficacy without significant side effects; however, neither safety nor efficacy endpoints were prespecified. Testing of such agents in formal trials, perhaps against sirolimus, should be undertaken to permit objective, quantitative assessment of outcomes and AEs before unregulated use becomes widespread.

\section{ELECTRONIC SUPPLEMENTARY MATERIAL}

The online version of this article (https://doi.org/10.1038/s41436018-0297-9) contains supplementary material, which is available to authorized users.

\section{ACKNOWLEDGEMENTS}

This research was supported by an Investigator-Initiated Research (IIR) Award from Pfizer (study ID WI189842), which involved provision of sirolimus. We thank patient support groups GoPI3Ks, MCM.net, and the CLOVES syndrome community for raising awareness of the study. The UK arm of the study was supported by the National Institute for Health Research (NIHR) through the Rare Disease Translational Research Collaboration and the Cambridge Biomedical Research Centre and by Wellcome Trust grant WT098498. The UK study was conducted on the NIHR/ Wellcome Trust Clinical Research Facility, Cambridge University Hospitals NHS Trust, Cambridge with the support of Cambridge Clinical Trials Unit (CCTU). K.M.K.-N., L.G.B., and J.C.S. are supported by intramural funding from the National Human Genome Research Institute (grant HG200388-03). K.Y.C. is supported by intramural funding from the National Institute of Diabetes and Digestive and Kidney Diseases (grant Z01 DK07013). The French arm of the study was supported by the Société Française de Dermatologie, Dijon University Hospital, Regional council of Burgundy (Plan d'Actions Régionales pour I'Innovation PARI 2015), the European Union through the PO FEDER-FSE Bourgogne 2014/2020 programs and FEDER 2016. The ideas and opinions expressed in this paper are those of the authors only and do not necessarily represent any position or policy of the National Institutes of Health or any other institution or organization with which any of the authors are affiliated.

\section{DISCLOSURE}

V.E.R.P. is an employee of Medlmmune Ltd and has stock holdings in AstraZeneca. L.G.B. and M.J.L. receive royalties from Genentech Corporation and L.G.B. is an advisor to the Illumina Corp. The other authors declare no conflicts of interest.

\section{REFERENCES}

1. Keppler-Noreuil KM, Rios JJ, Parker VER, et al. PIK3CA-related overgrowth spectrum (PROS): diagnostic and testing eligibility criteria, differential diagnosis, and evaluation. Am J Med Genet A. 2014;167A:287-295. 
2. Vanhaesebroeck B, Stephens L, Hawkins P. PI3K signalling: the path to discovery and understanding. Nat Rev Mol Cell Biol. 2012;13:195-203.

3. Kurek KC, Luks VL, Ayturk UM, et al. Somatic mosaic activating mutations in PIK3CA cause CLOVES syndrome. Am J Hum Genet. 2012;90:1108-1115.

4. Lindhurst MJ, Parker VER, Payne F, et al. Mosaic overgrowth with fibroadipose hyperplasia is caused by somatic activating mutations in PIK3CA. Nat Genet. 2012;44:928-933.

5. Rivière J-B, Mirzaa GM, O'Roak BJ, et al. De novo germline and postzygotic mutations in AKT3, PIK3R2 and PIK3CA cause a spectrum of related megalencephaly syndromes. Nat Genet. 2012;44:934-940.

6. Rios JJ, Paria N, Burns DK, et al. Somatic gain-of-function mutations in PIK3CA in patients with macrodactyly. Hum Mol Genet. 2013;22:444-451.

7. Hardwicke J, Khan MAA, Richards H, Warner RM, Lester R. Macrodactyly —options and outcomes. J Hand Surg Eur Vol. 2013;38:297-303.

8. Mirzaa G, Timms AE, Conti V, et al. PIK3CA-associated developmental disorders exhibit distinct classes of mutations with variable expression and tissue distribution. JCI Insight. 2016;1. pii: e87623

9. Keppler-Noreuil KM, Sapp JC, Lindhurst MJ, et al. Clinical delineation and natural history of the PIK3CA -related overgrowth spectrum. Am J Med Genet A. 2014;164:1713-1733.

10. Elkabets $M$, Vora $S$, Juric $D$, et al. mTORC1 inhibition is required for sensitivity to PI3Kp110 $\alpha$ inhibitors in PIK3CA-mutant breast cancer. Sci Transl Med. 2013;5:196ra99.

11. Loconte DC, Grossi V, Bozzao C, et al. Molecular and functional characterization of three different postzygotic mutations in PIK3CArelated overgrowth spectrum (PROS) patients: effects on PI3K/AKT/mTOR signaling and sensitivity to PIK3 inhibitors. PLoS ONE. 2015;10:e0123092.

12. Suzuki $Y$, Enokido $Y$, Yamada $K$, et al. The effect of rapamycin, NVPBEZ235, aspirin, and metformin on PI3K/AKT/mTOR signaling pathway of PIK3CA-related overgrowth spectrum (PROS). Oncotarget. 2017:8:45470-45483

13. Parker V, Huson S, Isaac I, et al. Sirolimus therapy for a patient with segmental overgrowth due to a mosaic activating mutation in phosphatidylinositol-3-kinase. Endocr Abstr. 2014;177:175-186.

14. Hammill AM, Wentzel M, Gupta A, et al. Sirolimus for the treatment of complicated vascular anomalies in children. Pediatr Blood Cancer. 2011;57:1018-1024.

15. Boscolo E, Limaye N, Huang L, et al. Rapamycin improves TIE2-mutated venous malformation in murine model and human subjects. J Clin Invest. 2015:125:3491-3504

16. Nadal M, Giraudeau B, Tavernier E, Jonville-Bera A, Lorette G, Maruani A. Efficacy and safety of mammalian target of rapamycin inhibitors in vascular anomalies: a systematic review. Acta Derm Venereol. 2016:96:448-452.

17. Adams DM, Trenor CC, Hammill AM, et al. Efficacy and safety of sirolimus in the treatment of complicated vascular anomalies. Pediatrics. 2016;137:e20153257-e20153257.

18. Kuentz P, St-Onge J, Duffourd $Y$, et al. Molecular diagnosis of PIK3CArelated overgrowth spectrum (PROS) in 162 patients and recommendations for genetic testing. Genet Med. 2017;19:989-997.
19. Scott JR, Courter JD, Saldaña SN, et al. Population pharmacokinetics of sirolimus in pediatric patients with neurofibromatosis type 1. Ther Drug Monit. 2013;35:332-337.

20. Parker VER, Knox RG, Zhang Q, Wakelam MJO, Semple RK. Phosphoinositide 3-kinase-related overgrowth: cellular phenotype and future therapeutic options. Lancet. 2015;385:S77.

21. Thomsen TK, Jensen VJ, Henriksen MG. In vivo measurement of human body composition by dual-energy X-ray absorptiometry (DXA). Eur J Surg. 1998;164:133-137.

22. Siri WE. Body composition from fluid spaces and density: analysis of methods. Nutrition. 1961;9:480-492

23. Skevington SM, Lotfy M, O'Connell KA, WHOQOL Group. The World Health Organization's WHOQOL-BREF quality of life assessment: psychometric properties and results of the international field trial. A report from the WHOQOL Group. Qual Life Res. 2004;13:299-310.

24. Varni JW, Seid M, Rode CA. The PedsQL: measurement model for the pediatric quality of life inventory. Med Care. 1999;37:126-139.

25. Varni JW, Burwinkle TM, Berrin SJ, et al. The PedsQL in pediatric cerebral palsy: reliability, validity, and sensitivity of the Generic Core Scales and Cerebral Palsy Module. Dev Med Child Neurol. 2006;48:442.

26. Varni JW, Seid M, Kurtin PS. PedsQL 4.0: reliability and validity of the Pediatric Quality of Life Inventory version 4.0 generic core scales in healthy and patient populations. Med Care. 2001;39:800-812.

27. Development of the World Health Organization WHOQOL-BREF quality of life assessment. The WHOOOL Group. Psychol Med. 1998:28:551-558.

28. Krueger DA, Care MM, Holland K, et al. Everolimus for subependymal giantcell astrocytomas in tuberous sclerosis. N Engl J Med. 2010;363:1801-1811.

29. CHMP. ANNEX I SUMMARY OF PRODUCT CHARACTERISTICS.http:// www.ema.europa.eu/ema/index.jsp?curl=pages/medicines/human/ medicines/000273/human_med_001010. jsp\&mid=WCOb01ac058001d124 Accessed 20 September 2018.

30. Venot Q, Blanc T, Rabia SH, et al. Targeted therapy in patients with PIK3CA-related overgrowth syndrome. Nature. 2018;558:540-546.

Open Access This article is licensed under a Creative Commons Attribution 4.0 International License, which permits use, sharing, adaptation, distribution and reproduction in any medium or format, as long as you give appropriate credit to the original author(s) and the source, provide a link to the Creative Commons license, and indicate if changes were made. The images or other third party material in this article are included in the article's Creative Commons license, unless indicated otherwise in a credit line to the material. If material is not included in the article's Creative Commons license and your intended use is not permitted by statutory regulation or exceeds the permitted use, you will need to obtain permission directly from the copyright holder. To view a copy of this license, visit http://creativecommons.org/licenses/ by/4.0\%.

(c) The Author(s) 2018

${ }^{1}$ Institute of Metabolic Science, University of Cambridge, Cambridge, UK. ${ }^{2}$ Medical Genomics and Metabolic Genetics Branch, National Human Genome Research Institute, National Institutes of Health, Bethesda, MD, USA. ${ }^{3}$ Centres de références Anomalies du Développement et Anomalies Dermatologiques Rares, Equipe GAD UMR1231 et FHU TRANSLAD, CHU Dijon-Bourgogne et Université de Bourgogne, Dijon, France. ${ }^{4}$ Centre d'Investigation Clinique INSERM 1432, Centre Hospitalier Universitaire de Dijon, Dijon, Bourgogne, France. ${ }^{5}$ The EMMES Corporation, Rockville, MD, USA. ${ }^{6}$ Section on Pediatric Diabetes and Metabolism, National Institute of Diabetes, Digestive, and Kidney Diseases, National Institutes of Health, Bethesda, MD, USA. ${ }^{7}$ Department of Dermatology, Uniformed Services University of the Health Sciences, Bethesda, MD, USA. ${ }^{8}$ Pharmacy Department, NIH Clinical Center, National Institutes of Health, Bethesda, MD, USA. ${ }^{9}$ Department of Dermatology and Reference Center for Genodermatoses and Rare Skin Diseases (MAGEC), Université Paris Descartes - Sorbonne Paris Cité, INSERM U1163, Institut Imagine, Institut Imagine, Hôpital Universitaire Necker-Enfants Malades, Paris, France. ${ }^{10}$ Department of Interventional Radiology, Dijon University Hospital, Dijon, France. ${ }^{11}$ Department of Dermatology, University Hospital Center of Angers, Angers, France. ${ }^{12}$ Department of Dermatology, Claude Bernard-Lyon 1 University and Hospices Civils de Lyon, Lyon, France. ${ }^{13} \mathrm{NCl}, \mathrm{CCR}$, Pediatric Oncology Branch, National Institutes of Health, Bethesda, MD, USA. ${ }^{14}$ Département de Génétique Médicale, Maladies Rares et Médecine Personnalisée, CHU de Montpellier, Montpellier, France. ${ }^{15}$ Service de Génétique médicale, Hôpital Jeanne de Flandre, CHRU de Lille, Lille, France. ${ }^{16}$ Centre for Cardiovascular Science, Queen's Medical Research Institute, University of Edinburgh, Edinburgh, UK 